\title{
Law of Large Numbers under Choquet Expectations
}

\author{
Jing Chen \\ School of Mathematics, Shandong University, Jinan 250100, China \\ Correspondence should be addressed to Jing Chen; chenjing07@hotmail.com \\ Received 13 December 2013; Accepted 13 January 2014; Published 2 March 2014 \\ Academic Editor: Litan Yan \\ Copyright (C) 2014 Jing Chen. This is an open access article distributed under the Creative Commons Attribution License, which \\ permits unrestricted use, distribution, and reproduction in any medium, provided the original work is properly cited. \\ With a new notion of independence of random variables, we establish the nonadditive version of weak law of large numbers (LLN) \\ for the independent and identically distributed (IID) random variables under Choquet expectations induced by 2-alternating \\ capacities. Moreover, we weaken the moment assumptions to the first absolute moment and characterize the approximate \\ distributions of random variables as well. Naturally, our theorem can be viewed as an extension of the classical LLN to the case \\ where the probability is no longer additive.
}

\section{Introduction}

Ever since the definition of capacity was introduced by Choquet [1] in 1954, it has been a heated scientific subject worldwide. Since in many application fields, such as finance, economics, and robust statistics, the traditional additive probability measures fail to provide adequate or good information to describe or interpret the uncertain phenomena accurately. Meanwhile, capacities, the nonadditive probability measures, seem to be a powerful tool to model the uncertainty when the assumption of additivity is suspect (e.g., Augustin [2], Maccheroni and Marinacci [3], Doob [4], and Schmeidler [5]).

In applied statistics and probabilities, law of large numbers (LLN) is one of the most frequently used results. Recently, many authors have investigated different kinds of LLNs for capacities. From 1999 to 2005, Maccheroni and Marinacci $[3,6]$ introduced the definition of independence of random variables relative to a capacity and then presented a strong LLN for totally monotone capacities. Around 2006, by adopting the partial differential equations (PDE), Peng [7-9] proved the LLN with the independence of random variables he defined under sublinear expectations. In 2009, Chareka [10] obtained the LLN for Choquet capacities by converting the nonadditive Choquet integral into the additive LebesgueStieltjes integral. Later, Chen [11] derived a natural extension of the classical Kolmogorov strong LLN to the subadditive case and the related application was given by Chen et al.
[12]. In 2011, using Chebyshev's inequality and Borel-Cantelli lemma for capacities, Li and Chen [13] provided the LLN for negatively correlated random variables. In 2013, Z. Chen and J. Chen [14] proposed a new proof of maximal distribution theorem and then derived the LLN under sublinear expectations with applications in finance. Most of the LLN literature above focuses on the estimation, or the confidence intervals of the average of random variables on a capacity space, only a few gives information of the distributions of random variables.

So far, the works closely related to our results are Peng [7] and Z. Chen and J. Chen [14] in the sense that the three LLNs are all proved from the perspective of convergence in distribution (in law). However, the substantive differences not only lie in assumptions, such as the definitions of independence and the moment conditions, but also lie in proving techniques: Peng achieves LLN with the help of PDE, while our LLN is proved by Feller's pure probabilistic method, using Taylor's expansions and some basic properties of Choquet expectations.

As we known, the key to prove classical LLN are the additivity of probabilities/expectations as well as the moment conditions of random variables. However, the Choquet expectations happen to be the nonadditive expectations. To overcome this nonadditive problem, we adopt the 2-alternating capacity; thus, the Choquet expectations induced by it turn to be subadditive ones, under which we consider the sequence $\left\{X_{i}\right\}_{i=1}^{\infty}$ of IID random variables converges in distribution. 
In this paper, firstly, we introduce a notion of independence of random variables under Choquet expectations, which is different from [7], then establish the nonadditive version of LLN, and characterize the approximate distribution of $(1 / n) \sum_{i=1}^{n} X_{i}$ for large $n$. Moreover, our moment condition is weaker than other Choquet literature's.

The paper is organized as follows. In Section 2, we present some basic concepts associated with the Choquet expectations. One of the most important ones is the new concept of independence we introduced in this paper, under which we establish our results. In Section 3, we state the LLN under Choquet expectations with proof followed by the conclusion remarks in Section 4.

\section{Preliminary}

In this section, we give an overview of the definitions and properties concerning capacities.

Let $(\Omega, \mathscr{F})$ be a measurable space. Suppose that $C_{b}(\mathbb{R})$ is the set of all bounded and continuous real-valued functions on $\mathbb{R}$ and $C_{b}^{2}(\mathbb{R})$ is the set of functions in $C_{b}(\mathbb{R})$ with bounded, continuous first- and second-order derivatives.

Definition 1. A set function $\mathbb{V}: \mathscr{F} \rightarrow[0,1]$ is called a capacity, if it satisfies the following properties:

(1) $\mathbb{V}(\emptyset)=0, \mathbb{V}(\Omega)=1$;

(2) $\mathbb{V}(A) \leq \mathbb{V}(B)$ whenever $A \subseteq B$ and $A, B \in \mathscr{F}$.

Definition 2. Let $X$ be a random variable on $(\Omega, \mathscr{F})$. The upper Choquet expectation (integral) of $X$ induced by a capacity $\mathbb{V}$ on $\mathscr{F}$ is defined by

$$
\begin{aligned}
\mathbb{C}_{V}[X]:= & \int_{\Omega} X d \mathbb{V}(t)=\int_{0}^{\infty} \mathbb{V}(X>t) d t \\
& +\int_{-\infty}^{0}[\mathbb{V}(X>t)-1] d t .
\end{aligned}
$$
by

The lower Choquet expectation of $X$ induced by $\mathbb{V}$ is given

$$
\mathscr{C}_{V}[X]:=-\mathbb{C}_{V}[-X], \quad \forall X \in \mathscr{F},
$$

which is conjugate to the upper expectation and satisfies $\mathscr{C}_{V}[X] \leq \mathbb{C}_{V}[X]$.

For simplicity, we can only consider the upper Choquet expectation in the sequel, since the lower (conjuagte) part can be considered similarly.

Lately, a few papers have discussed the Choquet expectation (integral); however, we only need the following basic properties in this paper.

Proposition 3 (see Dennerberg [15]). Let $X, Y$ be two random variables on $(\Omega, \mathscr{F})$, and let $\mathbb{C}_{V}$ be the upper Choquet expectation induced by a capacity $\mathbb{V}$. Then, one has

(1) monotonicity: $X \geq Y$ implies $\mathbb{C}_{V}[X] \geq \mathbb{C}_{V}[Y]$;

(2) positive homogeneity: $\mathbb{C}_{V}[\lambda X]=\lambda \mathbb{C}_{V}[X]$, for all $\lambda \geq$ 0 ;

(3) translation invariance: $\mathbb{C}_{V}[X+a]=\mathbb{C}_{V}[X]+a$, for all $a \in \mathbb{R}$.
Independence. We now recall the notion of independence in probability case briefly and then extend it to the nonadditive case. Let $\xi, \eta$ be two random variables on probability space $(\Omega, \mathscr{F}, P)$. Let $F_{\xi}$ and $F_{\eta}$ be the distribution functions of $\xi$ and $\eta$, respectively, under probability $P$; the expectation $E_{P}$ under additive $P$ is defined by (see Chung [16, page 45, Theorem 2.3])

$$
\begin{aligned}
E_{P}[\xi]= & \int_{-\infty}^{\infty} x d F_{\xi}(x) \\
= & \int_{0}^{\infty} P(\xi>x) d x \\
& +\int_{-\infty}^{0}[P(\xi>x)-1] d x .
\end{aligned}
$$

If $\xi$ and $\eta$ are said to be independent for any bounded realvalued function $\varphi$ on $\mathbb{R}$,

$$
\begin{aligned}
E_{P}[\varphi(\xi+\eta)] & =\int_{-\infty}^{\infty}\left(\int_{-\infty}^{\infty} \varphi(x+y) d F_{\eta}(y)\right) d F_{\xi}(x) \\
& =\int_{-\infty}^{\infty}\left(\int_{-\infty}^{\infty} \varphi(x+y) d F_{\xi}(x)\right) d F_{\eta}(y) .
\end{aligned}
$$

Then, the independence for additive expectation $E_{P}$ can be viewed as

$$
\begin{aligned}
E_{P}[\varphi(\xi+\eta)] & =E_{P}\left[E_{P}\left[\left.\varphi(x+\eta)\right|_{x=\xi}\right]\right] \\
& =E_{P}\left[E_{P}\left[\left.\varphi(\xi+y)\right|_{y=\eta}\right]\right] .
\end{aligned}
$$

It is worth pointing out that the independence above is mutual independence due to Fubini's theorem, but for nonadditive Choquet expectations, the mutuality is no longer true; that is, the second equality in (5) does not hold.

Motivated by the probability case, we give the definition of independence under Choquet expectations naturally.

Definition 4. Let $\mathbb{C}_{V}$ be the upper Choquet expectation, $X, Y, X_{i} \in \mathscr{F}, i \geq 1$. Then we have the following.

(i) Independence: random variable $Y$ is independent of $X$, if for any function $\varphi \in C_{b}(\mathbb{R})$,

$$
\mathbb{C}_{V}[\varphi(X+Y)]=\mathbb{C}_{V}\left[\mathbb{C}_{V}\left[\left.\varphi(x+Y)\right|_{x=X}\right]\right] .
$$

(ii) Identical distribution: random variables $X$ and $Y$ are said to be identically distributed, if for each realvalued function $\varphi$ with $\varphi(X), \varphi(Y) \in \mathscr{F}$,

$$
\mathbb{C}_{V}[\varphi(X)]=\mathbb{C}_{V}[\varphi(Y)]<\infty .
$$

(iii) IID sequence: a sequence $\left\{X_{i}\right\}_{i=1}^{\infty}$ of random variables is called independent and identically distributed (IID), if $X_{i}$ and $X_{j}$ are identically distributed for each $i, j \geqslant 1$ and $X_{i+1}$ is independent of $\sum_{j=1}^{i} X_{j}$.

Motivated by Peng [7], we give the notions of distribution functions and convergence in distribution for Choquet's case. 
Definition 5. Let $\mathbb{C}_{V}$ be the upper Choquet expectation induced by a capacity $\mathbb{V}$ on $\mathscr{F}, X \in \mathscr{F}$. For any real-valued function $\varphi$ on $\mathbb{R}$ with $\varphi(X) \in \mathscr{F}$, the distribution function of $X$ is defined by

$$
\mathbb{F}_{X}[\varphi]:=\mathbb{C}_{V}[\varphi(X)], \quad \forall X \in \mathscr{F} .
$$

Definition 6. A sequence $\left\{X_{i}\right\}_{i=1}^{\infty}$ of random variables is said to converge in distribution (in law) under upper Choquet expectation $\mathbb{C}_{V}$ on $\mathscr{F}$, if for each real-valued function $\varphi$ on $\mathbb{R}$ with $\varphi\left(X_{i}\right) \in \mathscr{F}, i \geq 1$, the sequence $\left\{\mathbb{C}_{V}\left[\varphi\left(X_{i}\right)\right]\right\}_{i=1}^{\infty}$ converges.

2-Alternating Capacities. A lot of work on capacities has focused on the 2-alternating case, since the 2-alternating capacities can be applied in many fields, for example, in game theory as certain convex games (see Shapley [17]), in Bayesian robustness [18], and so forth.

Definition 7. A capacity $\mathbb{V}$ is 2-alternating if for all $A, B \in \mathscr{F}$,

$$
\mathbb{V}(A \cup B) \leq \mathbb{V}(A)+\mathbb{V}(B)-\mathbb{V}(A \cap B) .
$$

The following proposition and lemma help us overcome the problem of nonadditivity of Choquet expectations.

Proposition 8 (see Dennerberg [15]). Let $(\Omega, \mathscr{F})$ be a measurable space, and let $\mathbb{V}$ be a 2-alternating capacity defined on $\mathscr{F}$. Then $\mathbb{V}$ is subadditive:

$$
\mathbb{V}\left(\bigcup_{i=1}^{n} A_{i}\right) \leq \sum_{i=1}^{n} \mathbb{V}\left(A_{i}\right), \quad \forall A_{i} \in \mathscr{F}, i \geq 1 .
$$

Lemma 9. Let $\mathbb{C}_{V}$ be the upper Choquet expectation induced by 2-alternating capacity $\mathbb{V}$. For any $X, Y \in \mathscr{F}$, one has the following.

(1) Subadditivity: $\mathbb{C}_{V}[X+Y] \leq \mathbb{C}_{V}[X]+\mathbb{C}_{V}[Y]$.

(2) For any constant $a \in \mathbb{R}$,

$$
\mathbb{C}_{V}[a X]=a^{+} \mathbb{C}_{V}[X]-a^{-} \mathscr{C}_{V}[X]
$$

$$
\text { where } a^{+}=\max \{a, 0\} \text { and } a^{-}=\max \{-a, 0\} .
$$

(3) Sublinearity: $-\mathbb{C}_{V}[|Y|] \leq \mathscr{C}_{V}[Y] \leq \mathbb{C}_{V}[X+Y]-$ $\mathbb{C}_{V}[X] \leq \mathbb{C}_{V}[Y] \leq \mathbb{C}_{V}[|Y|]$.

Proof. (1) is from Dennerberg [15]; (2) and (3) follow from (1) and Proposition 3.

\section{Main Results}

In this section, we first introduce three lemmas which we will make use of and then state the main theorem with proof.

Lemma 10. Let $\left\{X_{i}\right\}_{i=1}^{\infty}$ be a sequence of independent random variables on $(\Omega, \mathscr{F})$. Let $\mathbb{V}$ be a 2-alternating capacity defined on $\mathscr{F}$, and let $\mathbb{C}_{V}, \mathscr{C}_{V}$ be the induced upper, lower Choquet expectations, respectively. Then for any $\varphi \in C_{b}(\mathbb{R})$ and any constant $y_{i} \in \mathbb{R}$,

$$
I_{1} \leq \mathbb{C}_{V}\left[\varphi\left(\sum_{i=1}^{n} X_{i}\right)\right]-\varphi\left(\sum_{i=1}^{n} y_{i}\right) \leq I_{2},
$$

where

$$
\begin{aligned}
& I_{1}:=\sum_{m=1}^{n} \inf _{x \in \mathbb{R}}\left\{\mathbb{C}_{V}\left[\varphi\left(x+X_{n-(m-1)}-y_{m}\right)\right]-\varphi(x)\right\}, \\
& I_{2}:=\sum_{m=1}^{n} \sup _{x \in \mathbb{R}}\left\{\mathbb{C}_{V}\left[\varphi\left(x+X_{n-(m-1)}-y_{m}\right)\right]-\varphi(x)\right\} .
\end{aligned}
$$

Proof. Set $S_{n}:=\sum_{i=1}^{n} X_{i}, S_{0}=0$,

$$
\mathbb{C}_{V}\left[\varphi\left(S_{n}\right)\right]-\varphi\left(\sum_{i=1}^{n} y_{i}\right)=\sum_{m=0}^{n-1} \Delta_{m}
$$

where

$$
\begin{aligned}
\Delta_{m}:= & \mathbb{C}_{V}\left[\varphi\left(S_{n-m}+\sum_{i=1}^{m} y_{i}\right)\right] \\
& -\mathbb{C}_{V}\left[\varphi\left(S_{n-(m+1)}+\sum_{i=1}^{m+1} y_{i}\right)\right] .
\end{aligned}
$$

We now estimate the term $\Delta_{m}$ for $0 \leq m \leq n-1$.

Let $h(x):=\mathbb{C}_{V}\left[\varphi\left(x+X_{n-m}\right)\right]$, by the independence of $\left\{X_{i}\right\}_{i=1}^{n}$,

$$
\begin{aligned}
\mathbb{C}_{V}[\varphi & \left.\left(S_{n-m}+\sum_{i=1}^{m} y_{i}\right)\right] \\
& =\mathbb{C}_{V}\left[\left.\mathbb{C}_{V}\left[\varphi\left(x+X_{n-m}\right)\right]\right|_{x=S_{n-(m+1)}+\sum_{i=1}^{m} y_{i}}\right] \\
& =\mathbb{C}_{V}\left[h\left(S_{n-(m+1)}+\sum_{i=1}^{m} y_{i}\right)\right] .
\end{aligned}
$$

This with the sublinearity of $\mathbb{C}_{V}$ in Lemma 9 , for one side, implies,

$$
\begin{aligned}
\Delta_{m}= & \mathbb{C}_{V}\left[h\left(S_{n-(m+1)}+\sum_{i=1}^{m} y_{i}\right)\right] \\
& -\mathbb{C}_{V}\left[\varphi\left(S_{n-(m+1)}+\sum_{i=1}^{m} y_{i}+y_{m+1}\right)\right] \\
\leq & \mathbb{C}_{V}\left[h\left(S_{n-(m+1)}+\sum_{i=1}^{m} y_{i}\right)\right. \\
\leq & \left.-\varphi\left(S_{n-(m+1)}+\sum_{i=1}^{m} y_{i}+y_{m+1}\right)\right] \\
\leq & \sup _{x \in \mathbb{R}}\left\{h(x)-\varphi\left(x+y_{m+1}\right)\right\} \\
= & \sup _{x \in \mathbb{R}}\left\{\mathbb{C}_{V}\left[\varphi\left(x+X_{n-m}\right)\right]-\varphi\left(x+y_{m+1}\right)\right\} .
\end{aligned}
$$


For the other side,

$$
\begin{aligned}
& \Delta_{m}= \mathbb{C}_{V}\left[h\left(S_{n-(m+1)}+\sum_{i=1}^{m} y_{i}\right)\right] \\
&-\mathbb{C}_{V}\left[\varphi\left(S_{n-(m+1)}+\sum_{i=1}^{m} y_{i}+y_{m+1}\right)\right] \\
& \geq \mathscr{C}_{V}\left[h\left(S_{n-(m+1)}+\sum_{i=1}^{m} y_{i}\right)\right. \\
& \geq \inf _{x \in \mathbb{R}}\left\{h(x)-\varphi\left(x+y_{m+1}\right)\right\} \\
&=\inf _{x \in \mathbb{R}}\left\{\mathbb{C}_{V}\left[\varphi\left(x+X_{n-m}\right)\right]-\varphi\left(x+y_{m+1}\right)\right\} .
\end{aligned}
$$

That is,

$$
\begin{aligned}
\inf _{x \in \mathbb{R}} & \left\{\mathbb{C}_{V}\left[\varphi\left(x+X_{n-m}\right)\right]-\varphi\left(x+y_{m+1}\right)\right\} \\
& \leq \Delta_{m} \leq \sup _{x \in \mathbb{R}}\left\{\mathbb{C}_{V}\left[\varphi\left(x+X_{n-m}\right)\right]-\varphi\left(x+y_{m+1}\right)\right\} .
\end{aligned}
$$

This with (14) implies that

$$
\begin{aligned}
\sum_{m=0}^{n-1} \inf _{x \in \mathbb{R}} & \left\{\mathbb{C}_{V}\left[\varphi\left(x+X_{n-m}\right)\right]-\varphi\left(x+y_{m+1}\right)\right\} \\
& \leq \mathbb{C}_{V}\left[\varphi\left(S_{n}\right)\right]-\varphi\left(\sum_{i=1}^{n} y_{i}\right) \\
& \leq \sum_{m=0}^{n-1} \sup _{x \in \mathbb{R}}\left\{\mathbb{C}_{V}\left[\varphi\left(x+X_{n-m}\right)\right]-\varphi\left(x+y_{m+1}\right)\right\} .
\end{aligned}
$$

It then follows by the fact that

$$
\begin{aligned}
& \sup _{x \in \mathbb{R}}\left\{\mathbb{C}_{V}\left[\varphi\left(x+X_{n-m}\right)\right]-\varphi\left(x+y_{m+1}\right)\right\} \\
&=\sup _{x \in \mathbb{R}}\left\{\mathbb{C}_{V}\left[\varphi\left(x+X_{n-m}-y_{m+1}\right)\right]-\varphi(x)\right\} \\
& \inf _{x \in \mathbb{R}}\left\{\mathbb{C}_{V}\left[\varphi\left(x+X_{n-m}\right)\right]-\varphi\left(x+y_{m+1}\right)\right\} \\
&=\inf _{x \in \mathbb{R}}\left\{\mathbb{C}_{V}\left[\varphi\left(x+X_{n-m}-y_{m+1}\right)\right]-\varphi(x)\right\} .
\end{aligned}
$$

We complete the proof of this lemma.

Lemma 11. Let $\mathbb{V}$ be a 2-alternating capacity and $\mathbb{C}_{V}, \mathscr{C}_{V}$ be the induced upper, lower Choquet expectations, respectively. Let $\left\{X_{i}\right\}_{i=1}^{\infty}$ be a sequence of IID random variables with $\mathbb{C}_{V}\left[X_{i}\right]=\bar{\mu}$ and $\mathscr{C}_{V}\left[X_{i}\right]=\mu$ satisfying that for $i \geq 1$,

$$
\mathbb{C}_{V}\left[\left|X_{i}\right|\right]<\infty \text {. }
$$

Then, for each function $\varphi \in C_{b}^{2}(\mathbb{R})$, there exists a positive constant $b_{n}(\epsilon)$ with $b_{n}(\epsilon) \rightarrow 0$, as $n \rightarrow \infty$, such that

$$
\begin{array}{r}
\sum_{i=1}^{n} \sup _{x \in \mathbb{R}}\left\{\mathbb{C}_{V}\left[\varphi\left(x+\frac{X_{i}}{n}\right)\right]-\varphi(x)\right\} \\
\leq \sup _{x \in \mathbb{R}} G\left(\varphi^{\prime}(x), \bar{\mu}, \underline{\mu}\right)+b_{n}(\epsilon) .
\end{array}
$$

(2)

$$
\begin{aligned}
& \sum_{i=1}^{n} \inf _{x \in \mathbb{R}}\left\{\mathbb{C}_{V}\left[\varphi\left(x+\frac{X_{i}}{n}\right)\right]-\varphi(x)\right\} \\
& \geq \inf _{x \in \mathbb{R}} G\left(\varphi^{\prime}(x), \bar{\mu}, \underline{\mu}\right)-b_{n}(\epsilon),
\end{aligned}
$$

where $G(x, \bar{\mu}, \underline{\mu}):=x^{+} \bar{\mu}-x^{-} \underline{\mu}$.

Proof. Applying the Taylor expansion for function $\varphi, 0 \leq \theta_{1} \leq$ 1 ,

$$
\varphi\left(x+\frac{X_{i}}{n}\right)-\varphi(x)=\varphi^{\prime}(x) \frac{X_{i}}{n}+J_{n}\left(x, X_{i}\right),
$$

where $J_{n}\left(x, X_{i}\right):=\left(\varphi^{\prime}\left(x+\theta_{1}\left(X_{i} / n\right)\right)-\varphi^{\prime}(x)\right)\left(X_{i} / n\right)$.

Set the upper Choquet expectation $\mathbb{C}_{V}$ on both sides of the above equality, by the sublinearity of $\mathbb{C}_{V}$,

$$
\begin{aligned}
-\mathbb{C}_{V}\left[\left|J_{n}\left(x, X_{i}\right)\right|\right] \leq & \mathbb{C}_{V}\left[\varphi\left(x+\frac{X_{i}}{n}\right)-\varphi(x)\right] \\
& -\mathbb{C}_{V}\left[\varphi^{\prime}(x) \frac{X_{i}}{n}\right] \leq \mathbb{C}_{V}\left[\left|J_{n}\left(x, X_{i}\right)\right|\right] .
\end{aligned}
$$

Since $\mathbb{C}_{V}\left[X_{i}\right]=\bar{\mu}, \mathscr{C}_{V}\left[X_{i}\right]=\underline{\mu}, i=1,2, \ldots, n$, thus

$$
\mathbb{C}_{V}\left[\varphi^{\prime}(x) \frac{X_{i}}{n}\right]=\frac{1}{n} G\left(\varphi^{\prime}(x), \bar{\mu}, \underline{\mu}\right) .
$$

Therefore, by translation invariance of Proposition 3,

$$
\begin{aligned}
-\mathbb{C}_{V}\left[\left|J_{n}\left(x, X_{i}\right)\right|\right] \leq & \mathbb{C}_{V}\left[\varphi\left(x+\frac{X_{i}}{n}\right)\right]-\varphi(x) \\
& -\frac{1}{n} G\left(\varphi^{\prime}(x), \bar{\mu}, \underline{\mu}\right) \leq \mathbb{C}_{V}\left[\left|J_{n}\left(x, X_{i}\right)\right|\right] .
\end{aligned}
$$

Take supremum $\sup _{x \in \mathbb{R}}$ on both sides of (28),

$$
\begin{aligned}
\sup _{x \in \mathbb{R}}\{ & \left.-\mathbb{C}_{V}\left[\left|J_{n}\left(x, X_{i}\right)\right|\right]\right\} \\
& \leq \sup _{x \in \mathbb{R}}\left\{\mathbb{C}_{V}\left[\varphi\left(x+\frac{X_{i}}{n}\right)\right]-\varphi(x)-\frac{1}{n} G\left(\varphi^{\prime}(x), \bar{\mu}, \underline{\mu}\right)\right\} \\
& \leq \sup _{x \in \mathbb{R}} \mathbb{C}_{V}\left[\left|J_{n}\left(x, X_{i}\right)\right|\right] .
\end{aligned}
$$

For writing convenience, denote

$$
T_{n}^{i}(x):=\mathbb{C}_{V}\left[\varphi\left(x+\frac{X_{i}}{n}\right)\right]-\varphi(x)-\frac{1}{n} G\left(\varphi^{\prime}(x), \bar{\mu}, \underline{\mu}\right) .
$$


From (29), we have

$$
\begin{aligned}
-\sum_{i=1}^{n} \sup _{x \in \mathbb{R}} \mathbb{C}_{V}\left[\left|J_{n}\left(x, X_{i}\right)\right|\right] & \leq \sum_{i=1}^{n} \sup _{x \in \mathbb{R}} T_{n}^{i}(x) \\
& \leq \sum_{i=1}^{n} \sup _{x \in \mathbb{R}} \mathbb{C}_{V}\left[\left|J_{n}\left(x, X_{i}\right)\right|\right] .
\end{aligned}
$$

In order to prove this lemma, we only need to estimate the term $\sum_{i=1}^{n} \sup _{x \in \mathbb{R}} \mathbb{C}_{V}\left[\left|J_{n}\left(x, X_{i}\right)\right|\right]$.

Indeed, since $\left\{X_{i}\right\}_{i=1}^{n}$ is identically distributed and $\mathbb{C}_{V}\left[\left|X_{1}\right|\right]<\infty$, by the definition of Choquet expectation, for any $\epsilon>0$, we have $\mathbb{C}_{V}\left[\left|X_{1}\right| I_{\left\{\left|X_{1}\right|>n \epsilon\right\}}\right] \rightarrow 0$ as $n \rightarrow \infty$. Thus, for any $\epsilon>0$,

$$
\begin{aligned}
& \sum_{i=1}^{n} \sup _{x \in \mathbb{R}} \mathbb{C}_{V}\left[\left|J_{n}\left(x, X_{i}\right)\right|\right] \\
& \leq \sum_{i=1}^{n}\left\{\sup _{x \in \mathbb{R}} \mathbb{C}_{V}\left[\left|J_{n}\left(x, X_{i}\right)\right| I_{\left\{\left|X_{i} / n\right|>\epsilon\right\}}\right]\right. \\
& \left.+\sup _{x \in \mathbb{R}} \mathbb{C}_{V}\left[\left|J_{n}\left(x, X_{i}\right)\right| I_{\left\{\left|X_{i} / n\right| \leq \epsilon\right\}}\right]\right\} \\
& \leq \sum_{i=1}^{n} \mathbb{C}_{V}\left[\left(\sup _{x \in \mathbb{R}}\left|\varphi^{\prime}\left(x+\theta_{1} \frac{X_{i}}{n}\right)\right|+\sup _{x \in \mathbb{R}}\left|\varphi^{\prime}(x)\right|\right)\right. \\
& \left.\times \frac{\left|X_{i}\right|}{n} I_{\left\{\left|X_{i}\right|>n \epsilon\right\}}\right] \\
& +\sum_{i=1}^{n} \frac{1}{2} \mathbb{C}_{V}\left[\sup _{x \in \mathbb{R}}\left|\varphi^{\prime \prime}\left(x+\theta_{2} \frac{X_{i}}{n}\right)\right| \frac{X_{i}^{2}}{n^{2}} I_{\left\{\left|X_{i}\right| \leq n \epsilon\right\}}\right] \\
& \leq \sum_{i=1}^{n} \frac{1}{n} 2\left\|\varphi^{\prime}\right\| \mathbb{C}_{V}\left[\left|X_{i}\right| I_{\left\{\left|X_{i}\right|>n \epsilon\right\}}\right] \\
& +\sum_{i=1}^{n} \frac{1}{n^{2}}\left\|\varphi^{\prime \prime}\right\| \mathbb{C}_{V}\left[\left|X_{i}\right|\right] n \epsilon \\
& =2\left\|\varphi^{\prime}\right\| \mathbb{C}_{V}\left[\left|X_{1}\right| I_{\left\{\left|X_{1}\right|>n \epsilon\right\}}\right]+\left\|\varphi^{\prime \prime}\right\| \mathbb{C}_{V}\left[\left|X_{1}\right|\right] \epsilon \\
& :=b_{n}(\epsilon) \longrightarrow 0, \quad n \longrightarrow \infty \text {, }
\end{aligned}
$$

where $\|\varphi\|:=\sup _{x \in \mathbb{R}}|\varphi(x)|$ and $0 \leq \theta_{2} \leq 1$.

Combining (31) and (32), for the arbitrary of $\epsilon$, as $n \rightarrow$ $\infty$, we have

$$
\sum_{i=1}^{n} \sup _{x \in \mathbb{R}} T_{n}^{i}(x) \leq \sum_{i=1}^{n} \sup _{x \in \mathbb{R}} \mathbb{C}_{V}\left[\left|J_{n}\left(x, X_{i}\right)\right|\right] \leq b_{n}(\epsilon) \longrightarrow 0 .
$$

(1) From (33), we have

$$
\begin{aligned}
\sum_{i=1}^{n} \sup _{x \in \mathbb{R}}\left\{\mathbb{C}_{V}\left[\varphi\left(x+\frac{X_{i}}{n}\right)\right]-\varphi(x)\right\} \\
=\sum_{i=1}^{n} \sup _{x \in \mathbb{R}}\left\{\mathbb{C}_{V}\left[\varphi\left(x+\frac{X_{i}}{n}\right)\right]-\varphi(x)\right. \\
\left.\quad-\frac{1}{n} G\left(\varphi^{\prime}(x), \bar{\mu}, \underline{\mu}\right)+\frac{1}{n} G\left(\varphi^{\prime}(x), \bar{\mu}, \underline{\mu}\right)\right\}
\end{aligned}
$$

$$
\begin{aligned}
& \leq \sum_{i=1}^{n} \sup _{x \in \mathbb{R}} T_{n}^{i}(x)+\sum_{i=1}^{n} \sup _{x \in \mathbb{R}}\left\{\frac{1}{n} G\left(\varphi^{\prime}(x), \bar{\mu}, \underline{\mu}\right)\right\} \\
& \leq b_{n}(\epsilon)+\sup _{x \in \mathbb{R}} G\left(\varphi^{\prime}(x), \bar{\mu}, \underline{\mu}\right) .
\end{aligned}
$$

(2) Taking infimum inf ${ }_{x \in \mathbb{R}}$ on both sides of (28), similar to the proof of (1), we have

$\sum_{i=1}^{n} \inf _{x \in \mathbb{R}} T_{n}^{i}(x) \geq-\sum_{i=1}^{n} \sup _{x \in \mathbb{R}} \mathbb{C}_{V}\left[\left|J_{n}\left(x, X_{i}\right)\right|\right] \geq-b_{n}(\epsilon)$.

Hence,

$$
\begin{aligned}
& \sum_{i=1}^{n} \inf _{x \in \mathbb{R}}\left\{\mathbb{C}_{V}\left[\varphi\left(x+\frac{X_{i}}{n}\right)\right]-\varphi(x)\right\} \\
& \geq \sum_{i=1}^{n} \inf _{x \in \mathbb{R}} T_{n}^{i}(x)+\sum_{i=1}^{n} \inf _{x \in \mathbb{R}}\left\{\frac{1}{n} G\left(\varphi^{\prime}(x), \bar{\mu}, \underline{\mu}\right)\right\} \\
& \geq-b_{n}(\epsilon)+\inf _{x \in \mathbb{R}} G\left(\varphi^{\prime}(x), \bar{\mu}, \underline{\mu}\right) .
\end{aligned}
$$

The proof of the lemma is complete.

Lemma 12. Let $G(x, y, z)$ be the function defined in Lemma 11; that is,

$$
G(x, y, z):=x^{+} y-x^{-} z
$$

Then

(1) $\inf _{y \in D_{n}} \sup _{x \in \mathbb{R}} G\left(\varphi^{\prime}(x), \bar{\mu}-(1 / n) \sum_{i=1}^{n} y_{i}, \underline{\mu}-(1 /\right.$ n) $\left.\sum_{i=1}^{n} y_{i}\right)=0$.

(2) $\inf _{y \in D_{n}} \inf _{x \in \mathbb{R}} G\left(\varphi^{\prime}(x), \bar{\mu}-(1 / n) \sum_{i=1}^{n} y_{i}, \underline{\mu}-(1 /\right.$ n) $\left.\sum_{i=1}^{n} y_{i}\right)=0$,

where $D_{n}:=\left\{y:=\left(y_{1}, y_{2}, \ldots, y_{n}\right): y_{i} \in[\mu, \bar{\mu}], i=\right.$ $1,2, \ldots, n\}$.

Proof. Consider the following:

$$
\begin{array}{r}
\inf _{y \in D_{n}} \sup _{x \in \mathbb{R}} G\left(\varphi^{\prime}(x), \bar{\mu}-\frac{1}{n} \sum_{i=1}^{n} y_{i}, \underline{\mu}-\frac{1}{n} \sum_{i=1}^{n} y_{i}\right) \\
=\inf _{y \in D_{n}} \sup _{x \in \mathbb{R}}\left\{\left(\varphi^{\prime}(x)\right)^{+}\left(\bar{\mu}-\frac{1}{n} \sum_{i=1}^{n} y_{i}\right)\right. \\
\left.-\left(\varphi^{\prime}(x)\right)^{-}\left(\underline{\mu}-\frac{1}{n} \sum_{i=1}^{n} y_{i}\right)\right\} \\
=\inf _{y \in D_{n}} \sup _{x \in \mathbb{R}}\left\{\left(\varphi^{\prime}(x)\right)^{+}\left(\bar{\mu}-\frac{1}{n} \sum_{i=1}^{n} y_{i}\right)\right. \\
\left.+\left(\varphi^{\prime}(x)\right)^{-}\left(\frac{1}{n} \sum_{i=1}^{n} y_{i}-\underline{\mu}\right)\right\} .
\end{array}
$$


Observing the above equality, obviously, if $\varphi^{\prime}(x) \geq 0$, note the fact that $(1 / n) \sum_{i=1}^{n} y_{i} \in[\mu, \bar{\mu}]$, therefore, the above equality is equal to

$$
\begin{aligned}
& \inf _{y \in D_{n}} \sup _{x \in \mathbb{R}}\left\{\left(\varphi^{\prime}(x)\right)^{+}\left(\bar{\mu}-\frac{1}{n} \sum_{i=1}^{n} y_{i}\right)\right\} \\
& =\sup _{x \in \mathbb{R}}\left(\varphi^{\prime}(x)\right)^{+} \inf _{y \in D_{n}}\left(\bar{\mu}-\frac{1}{n} \sum_{i=1}^{n} y_{i}\right)=0 .
\end{aligned}
$$

Similarly, if $\varphi^{\prime}(x)<0$, it is easy to prove that

$$
\begin{aligned}
& \inf _{y \in D_{n}} \sup _{x \in \mathbb{R}}\left\{\left(\varphi^{\prime}(x)\right)^{-}\left(\frac{1}{n} \sum_{i=1}^{n} y_{i}-\underline{\mu}\right)\right\} \\
& =\sup _{x \in \mathbb{R}}\left(\varphi^{\prime}(x)\right)^{-} \inf _{y \in D_{n}}\left(\frac{1}{n} \sum_{i=1}^{n} y_{i}-\underline{\mu}\right)=0 .
\end{aligned}
$$

The proof of (1) is complete. (2) can be proved in the same way.

Theorem 13 (Law of large number). Let $\mathbb{V}$ be a 2-alternating capacity defined on $\mathscr{F}$, and let $\mathbb{C}_{V}, \mathscr{C}_{V}$ be the induced upper, lower Choquet expectations, respectively. Let $\left\{X_{i}\right\}_{i=1}^{\infty}$ be a sequence of IID random variables on $(\Omega, \mathscr{F})$ with $\mathbb{C}_{V}\left[X_{i}\right]=\bar{\mu}$, $\mathscr{C}_{V}\left[X_{i}\right]=\underline{\mu}$. Assume that for $i \geq 1$,

$$
\mathbb{C}_{V}\left[\left|X_{i}\right|\right]<\infty .
$$

Then for each function $\varphi \in C_{b}^{2}(\mathbb{R})$,

(1)

$$
\lim _{n \rightarrow \infty} \mathbb{C}_{V}\left[\varphi\left(\frac{1}{n} \sum_{i=1}^{n} X_{i}\right)\right]=\sup _{\underline{\mu} \leq x \leq \bar{\mu}} \varphi(x)
$$

(2)

$$
\lim _{n \rightarrow \infty} \mathscr{C}_{V}\left[\varphi\left(\frac{1}{n} \sum_{i=1}^{n} X_{i}\right)\right]=\inf _{\underline{\mu} \leq x \leq \bar{\mu}} \varphi(x) .
$$

Proof. (1) Set partial sums $S_{n}:=\sum_{i=1}^{n} X_{i}, S_{0}=0$. Let $D_{n}$ be the set denoted in Lemma 12; that is,

$$
D_{n}:=\left\{y:=\left(y_{1}, y_{2}, \ldots, y_{n}\right): \underline{\mu} \leq y_{i} \leq \bar{\mu}, i=1, \ldots, n\right\} .
$$

Obviously,

$$
\sup _{y \in D_{n}} \varphi\left(\frac{1}{n} \sum_{i=1}^{n} y_{i}\right)=\sup _{\underline{\mu} \leq x \leq \bar{\mu}} \varphi(x) .
$$

Then,

$$
\begin{aligned}
\mathbb{C}_{V}\left[\varphi\left(\frac{1}{n} S_{n}\right)\right]-\sup _{\underline{\mu} \leq x \leq \bar{\mu}} \varphi(x) \\
=\mathbb{C}_{V}\left[\varphi\left(\frac{1}{n} S_{n}\right)\right]-\sup _{y \in D_{n}} \varphi\left(\frac{1}{n} \sum_{i=1}^{n} y_{i}\right) \\
=\inf _{y \in D_{n}}\left\{\mathbb{C}_{V}\left[\varphi\left(\frac{1}{n} S_{n}\right)\right]-\varphi\left(\frac{1}{n} \sum_{i=1}^{n} y_{i}\right)\right\} .
\end{aligned}
$$

By Lemma 10, Lemma 11(1), and Lemma 12(1), for any $\epsilon>0$,

$$
\begin{aligned}
& \inf _{y \in D_{n}}\left\{\mathbb{C}_{V}\left[\varphi\left(\frac{1}{n} S_{n}\right)\right]-\varphi\left(\frac{1}{n} \sum_{i=1}^{n} y_{i}\right)\right\} \\
& \leq \inf _{y \in D_{n}} \sum_{i=1}^{n} \sup _{x \in \mathbb{R}}\left\{\mathbb{C}_{V}\left[\varphi\left(x+\frac{X_{i}-y_{i}}{n}\right)\right]-\varphi(x)\right\} \\
& \leq \inf _{y \in D_{n}}\left\{\sup _{x \in \mathbb{R}} G\left(\varphi^{\prime}(x), \bar{\mu}-\frac{1}{n} \sum_{i=1}^{n} y_{i}, \underline{\mu}-\frac{1}{n} \sum_{i=1}^{n} y_{i}\right)+b_{n}(\epsilon)\right\} \\
& \quad=\inf _{y \in D_{n}} \sup _{x \in \mathbb{R}} G\left(\varphi^{\prime}(x), \bar{\mu}-\frac{1}{n} \sum_{i=1}^{n} y_{i}, \underline{\mu}-\frac{1}{n} \sum_{i=1}^{n} y_{i}\right)+b_{n}(\epsilon) \\
& \quad=b_{n}(\epsilon) \longrightarrow 0, \quad n \longrightarrow \infty .
\end{aligned}
$$

This with (46) implies that

$$
\limsup _{n \rightarrow \infty} \mathbb{C}_{V}\left[\varphi\left(\frac{1}{n} S_{n}\right)\right] \leq \sup _{\underline{\mu} \leq x \leq \bar{\mu}} \varphi(x) .
$$

Note that, similar to (32), $b_{n}(\epsilon)$ in (47) is still a positive constant with $b_{n}(\epsilon) \rightarrow 0$ as $n \rightarrow \infty$. To be specific, due to the following facts: (a) $\left|y_{i}\right| \leq|\bar{\mu}|+|\mu|$ for $y \in D_{n}$; (b) $\left\{X_{i}\right\}_{i=1}^{n}$ is identically distributed; (c) by the definition of Choquet expectation, $\mathbb{C}_{V}\left[\left|X_{1}\right|\right]<\infty$ implies $\mathbb{C}_{V}\left[\left|X_{1}\right| I_{\left\{\left|X_{1}\right|>n \epsilon-|\bar{\mu}|-|\mu|\right\}}\right] \rightarrow 0$ as $n \rightarrow \infty$; and (d) $\mathbb{C}_{V}\left[I_{\left\{\left|X_{1}\right|>n \epsilon-|\bar{\mu}|-|\mu|\right\}}\right] \leq(1 /(n \bar{\varepsilon}-|\bar{\mu}|-|\underline{\mu}|)) \mathbb{C}_{V}\left[\left|X_{1}\right|\right] \rightarrow 0$ as $n \rightarrow \infty$. Then for any $\epsilon>0$, we have

$$
\begin{aligned}
& \sum_{i=1}^{n} \sup _{x \in \mathbb{R}} \mathbb{C}_{V}\left[\left|J_{n}\left(x, X_{i}-y_{i}\right)\right|\right] \\
& \leq \sum_{i=1}^{n}\left\{\frac{1}{n} 2\left\|\varphi^{\prime}\right\| \mathbb{C}_{V}\left[\left|X_{i}-y_{i}\right| I_{\left\{\left|X_{i}-y_{i}\right|>n \epsilon\right\}}\right]\right. \\
& \left.+\frac{1}{n}\left\|\varphi^{\prime \prime}\right\| \mathbb{C}_{V}\left[\left|X_{i}-y_{i}\right|\right] \epsilon\right\} \\
& \leq 2\left\|\varphi^{\prime}\right\| \frac{1}{n} \sum_{i=1}^{n}\left\{\mathbb { C } _ { V } \left[\left|X_{i}\right| I_{\left.\left\{\left|X_{i}\right|>n \epsilon-|\bar{\mu}|-|\underline{\mu}|\right\}\right]}\right.\right. \\
& +(|\bar{\mu}|+|\underline{\mu}|) \mathbb{C}_{V}\left[I_{\left.\left.\left\{\left|X_{i}\right|>n \epsilon-|\bar{\mu}|-|\underline{\mu}|\right\}\right]\right\}}\right] \\
& +\epsilon\left\|\varphi^{\prime \prime}\right\| \frac{1}{n} \sum_{i=1}^{n}\left(\mathbb{C}_{V}\left[\left|X_{i}\right|\right]+|\bar{\mu}|+|\underline{\mu}|\right) \\
& =2\left\|\varphi^{\prime}\right\|\left\{\mathbb{C}_{V}\left[\left|X_{1}\right| I_{\left.\left\{\left|X_{1}\right|>n \epsilon-|\bar{\mu}|-|\underline{\mu}|\right\}\right]}\right]\right. \\
& \quad+(|\bar{\mu}|+|\underline{\mu}|) \mathbb{C}_{V}\left[I_{\left.\left.\left\{\left|X_{1}\right|>n \epsilon-|\bar{\mu}|-|\underline{\mu}|\right\}\right]\right\}}\right] \\
& +\epsilon\left\|\varphi^{\prime \prime}\right\|\left(\mathbb{C}_{V}\left[\left|X_{1}\right|\right]+|\bar{\mu}|+|\underline{\mu}|\right) \\
& :=b_{n}(\epsilon) \longrightarrow 0, \quad n \longrightarrow \infty .
\end{aligned}
$$


On the other hand, by Lemma 10, Lemma 11(2), and Lemma 12(2), for any $\epsilon>0$,

$$
\begin{aligned}
& \inf _{y \in D_{n}}\left\{\mathbb{C}_{V}\left[\varphi\left(\frac{1}{n} S_{n}\right)\right]-\varphi\left(\frac{1}{n} \sum_{i=1}^{n} y_{i}\right)\right\} \\
& \geq \inf _{y \in D_{n}} \sum_{n=1}^{n} \inf _{x \in \mathbb{R}}\left\{\mathbb{C}_{V}\left[\varphi\left(x+\frac{X_{i}-y_{i}}{n}\right)\right]-\varphi(x)\right\} \\
& \geq \inf _{y \in D_{n}}\left\{\inf _{x \in \mathbb{R}} G\left(\varphi^{\prime}(x), \bar{\mu}-\frac{1}{n} \sum_{i=1}^{n} y_{i}, \underline{\mu}-\frac{1}{n} \sum_{i=1}^{n} y_{i}\right)-b_{n}(\epsilon)\right\} \\
& \quad=\inf _{y \in D_{n}} \inf _{x \in \mathbb{R}} G\left(\varphi^{\prime}(x), \bar{\mu}-\frac{1}{n} \sum_{i=1}^{n} y_{i}, \underline{\mu}-\frac{1}{n} \sum_{i=1}^{n} y_{i}\right)-b_{n}(\epsilon) \\
& =-b_{n}(\epsilon) \longrightarrow 0, \quad n \longrightarrow \infty .
\end{aligned}
$$

Together with (46), we have

$$
\liminf _{n \rightarrow \infty} \mathbb{C}_{V}\left[\varphi\left(\frac{1}{n} S_{n}\right)\right] \geq \sup _{\underline{\mu} \leq x \leq \bar{\mu}} \varphi(x) .
$$

Combining (48) and (51), (42) is achieved. Hence, we obtain the LLN under upper Choquet expectation $\mathbb{C}_{V}$ induced by the 2-alternating capacity $\mathbb{V}$.

(2) Since the following conjugate property, for $i \geq 1$,

$$
\mathscr{C}_{V}\left[\varphi\left(X_{i}\right)\right]=-\mathbb{C}_{V}\left[-\varphi\left(X_{i}\right)\right], \quad \forall X_{i} \in \mathscr{F},
$$

the part for lower Choquet expectation $\mathscr{C}_{V}$ can be proved similarly, we just omit it.

Remark 14. The condition $\varphi \in C_{b}^{2}(\mathbb{R})$ in Theorem 13 can be weaken to $\varphi \in C_{b}(\mathbb{R})$. Further, the IID condition can be weaken to "the independent random variables satisfying the Choquet absolute moment condition"; see our future work.

\section{Conclusion Remarks}

In summary, under the new concept of independence, we propose the nonadditive version of LLN under Choquet expectations induced by 2 -alternating capacities and characterize the approximate distributions of random variables. Meanwhile, we weaken the moment conditions to the first absolute moment condition compared to other Choquet LLNs', such as Chareka [10]. Our main technique is purely probabilistic and elementary, thus, it can be viewed as a natural extension of the traditional LLN to the case where the probability is no longer additive.

\section{Conflict of Interests}

The author declares that there is no conflict of interests regarding the publication of this paper.

\section{Acknowledgments}

The author thanks Professor Zengjing Chen and Dr. Xiaoyan Chen for their valuable suggestions. This research is supported by the National Natural Science Foundation of China (no. 11231005).

\section{References}

[1] G. Choquet, “Theory of capacities," Annales de l'Institut Fourier, vol. 5, pp. 131-295, 1954.

[2] T. Augustin, "Optimal decisions under complex uncertaintybasic notions and a general algorithm for data-based decision making with partial prior knowledge described by interval probability," Gesellschaft für Angewandte Mathematik und Mechanik, vol. 84, no. 10-11, pp. 678-687, 2004.

[3] F. Maccheroni and M. Marinacci, "A strong law of large numbers for capacities," The Annals of Probability, vol. 33, no. 3, pp. 11711178, 2005.

[4] J. L. Doob, Classical Potential Theory and Its Probabilistic Counterpart, vol. 262, Springer, New York, NY, USA, 1984.

[5] D. Schmeidler, "Subjective probability and expected utility without additivity," Econometrica, vol. 57, no. 3, pp. 571-587, 1989.

[6] M. Marinacci, "Limit laws for non-additive probabilities and their frequentist interpretation," Journal of Economic Theory, vol. 84, no. 2, pp. 145-195, 1999.

[7] S. Peng, "Law of large number and central limit theorem under nonlinear expectations," 2007, http://arxiv.org/abs/math/ 0702358vl.

[8] S. Peng, "A new central limit theorem under sublinear expectations," 2008, http://arxiv.org/abs/0803.2656.

[9] S. Peng, "Survey on normal distributions, central limit theorem, Brownian motion and the related stochastic calculus under sublinear expectations," Science in China, vol. 52, no. 7, pp. 13911411, 2009.

[10] P. Chareka, “The central limit theorem for capacities," Statistics \& Probability Letters, vol. 79, no. 12, pp. 1456-1462, 2009.

[11] Z. Chen, "The strong law of large numbers for capacities," 2010, http://arxiv.org/abs/1006.0749.

[12] Z. Chen, P. Wu, and B. Li, "A strong law of large numbers for non-additive probabilities," International Journal of Approximate Reasoning, vol. 54, no. 3, pp. 365-377, 2013.

[13] W. Li and Z. Chen, "Laws of large numbers of negatively correlated random variables for capacities," Acta Mathematicae Applicatae Sinica, vol. 27, no. 4, pp. 749-760, 2011.

[14] Z. Chen and J. Chen, "A new proof of maximal distribution theorem," preprint, 2013.

[15] D. Dennerberg, Non-Additive Measure and Intergral, Kluwer Academic Publisher, Dordrecht, The Netherlands, 1994.

[16] K. L. Chung, A Course in Probability Theory, Academic Press, New York, NY, USA, 2nd edition, 1974.

[17] L. S. Shapley, "Cores of convex games," International Journal of Game Theory, vol. 1, pp. 11-26, 1971.

[18] L. A. Wasserman and J. B. Kadane, "Bayes' theorem for Choquet capacities," The Annals of Statistics, vol. 18, no. 3, pp. 1328-1339, 1990. 


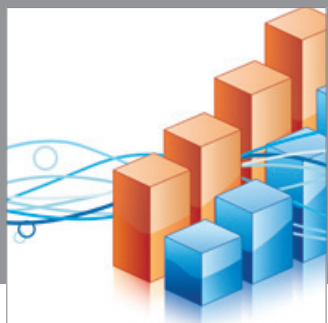

Advances in

Operations Research

mansans

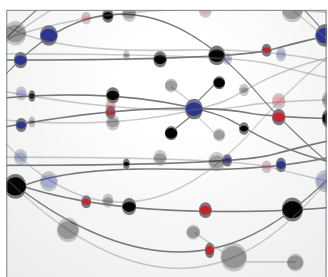

The Scientific World Journal
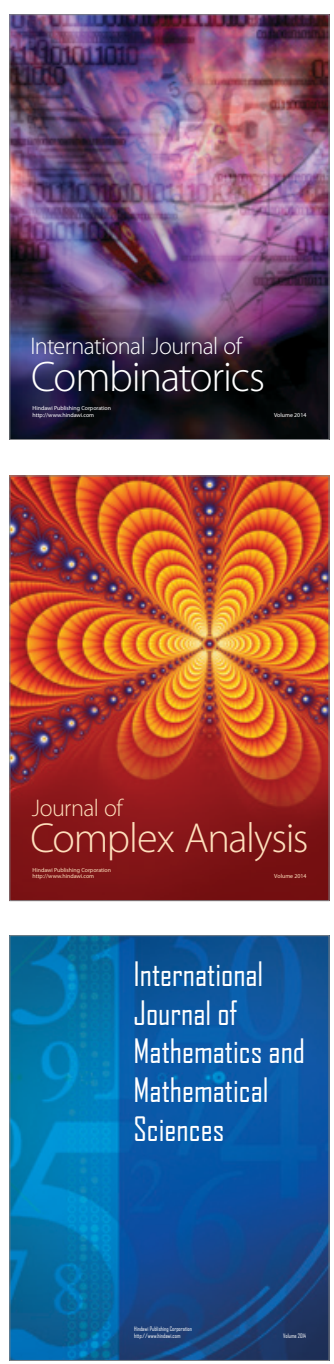
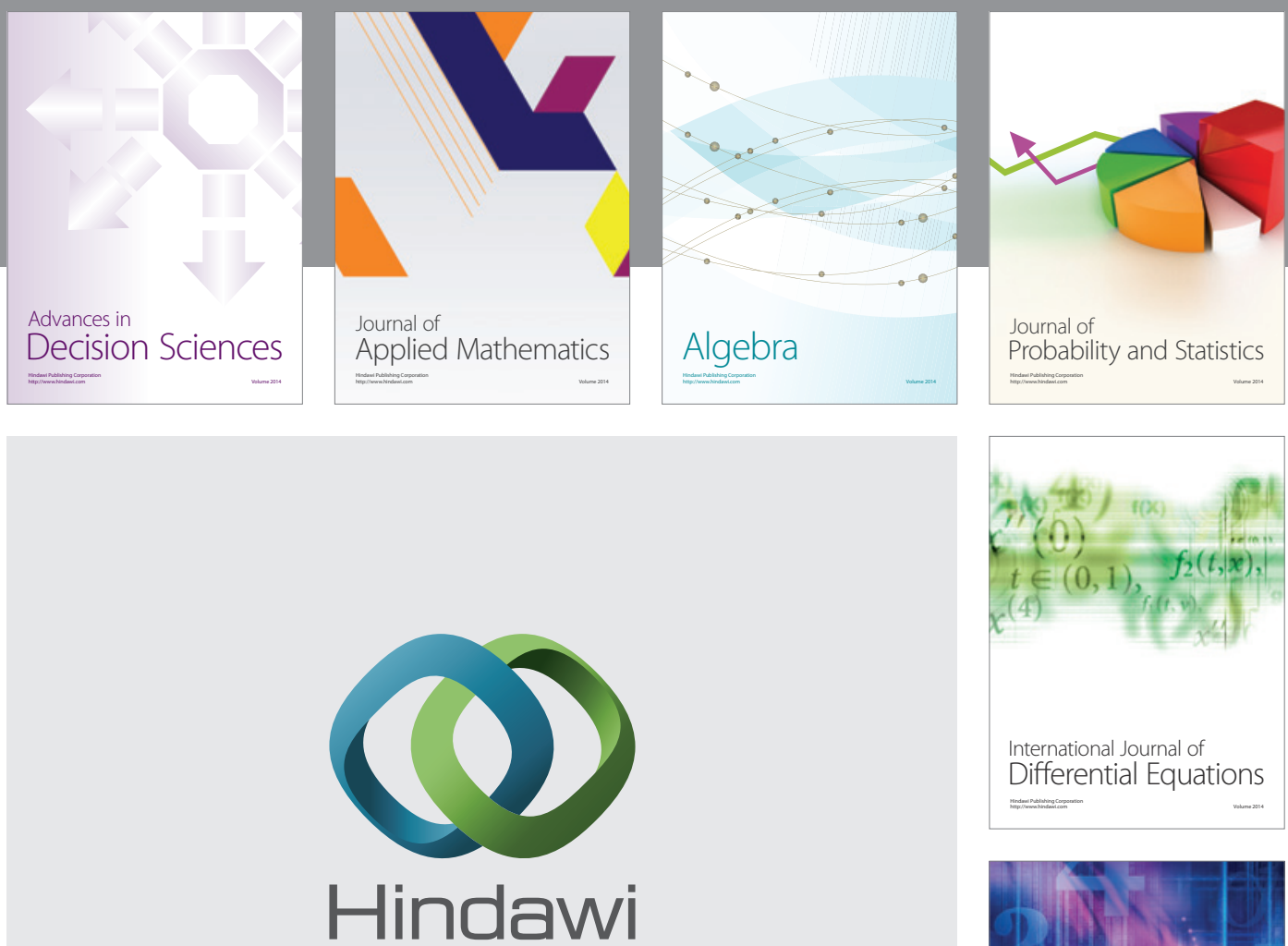

Submit your manuscripts at http://www.hindawi.com
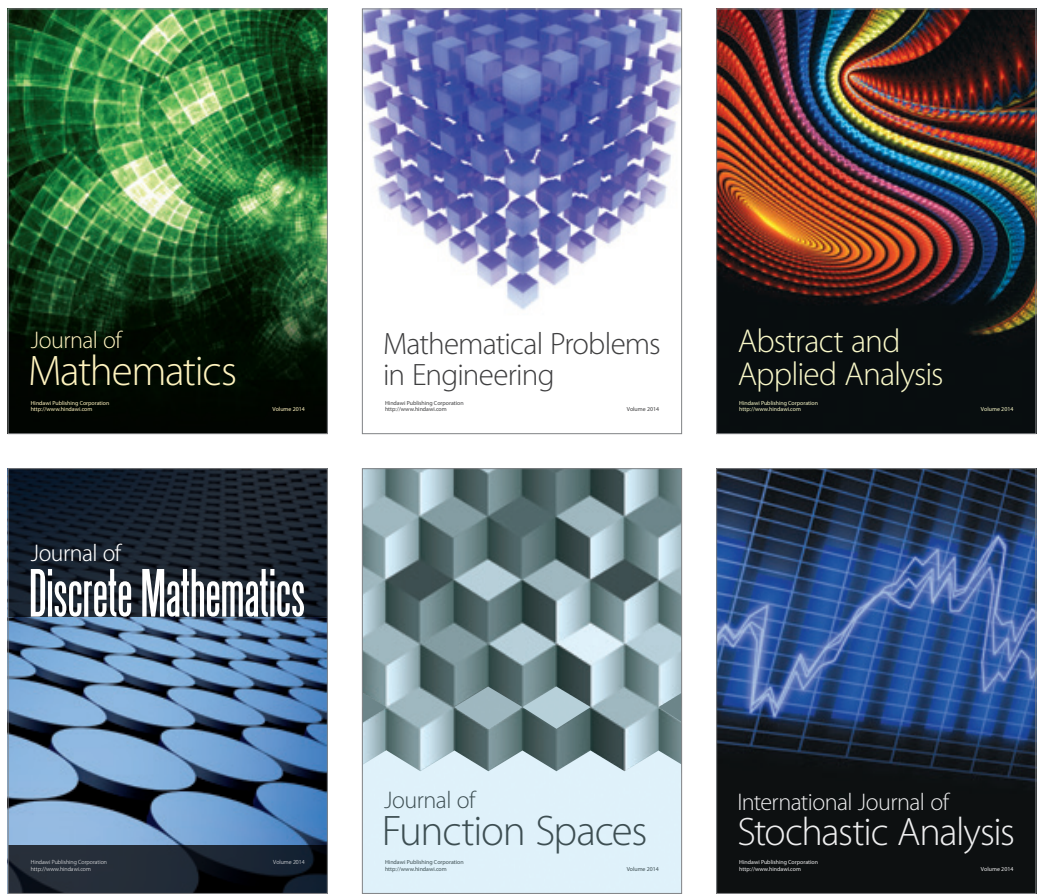

Journal of

Function Spaces

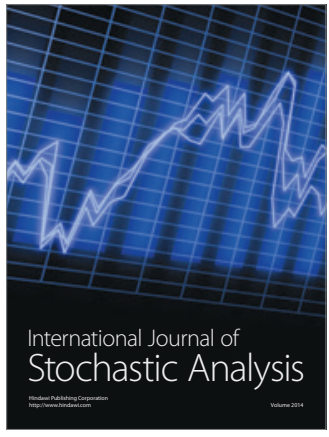

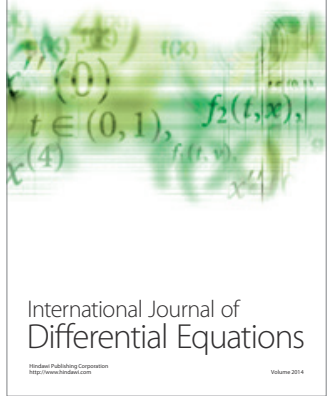
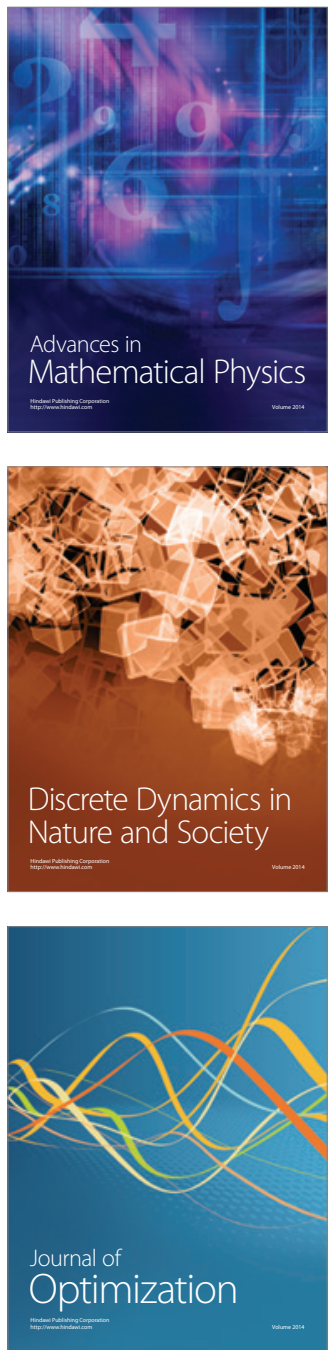